hep-th/0209090

\title{
Open String Creation by S-branes
}

\author{
Andrew Strominger \\ Department of Physics \\ Harvard University \\ Cambridge, MA 02138
}

\begin{abstract}
An sp-brane can be viewed as the creation and decay of an unstable $\mathrm{D}(\mathrm{p}+1)$-brane. It is argued that the decaying half of an sp-brane can be described by a variant of boundary Liouville theory. The pair creation of open strings by a decaying s-brane is studied in the minisuperspace approximation to the Liouville theory. In this approximation a Hagedornlike divergence is found in the pair creation rate, suggesting the s-brane energy is rapidly transferred into closed string radiation.
\end{abstract}

Talk presented at the String 2002 Conference, August 12-15, Hangzhou, China 


\section{Contents}

1. Introduction . . . . . . . . . . . . . . . . . . . . . . . . . . . . . . . . . 1

2. Scalar Field with Time-Dependent Mass . . . . . . . . . . . . . . . . . . . . . 3

2.1. Exponential Mass . . . . . . . . . . . . . . . . . . . . . . . . . . 3

2.2. Hyperbolic Mass . . . . . . . . . . . . . . . . . . . . . . . . . . . . . .

2.3. Particle Creation . . . . . . . . . . . . . . . . . . . . . . . . . . . . . 4

3. Minisuperspace Approximation . . . . . . . . . . . . . . . . . . . . . . . . . 5

4. The Half-s-brane and Boundary Liouville Theory . . . . . . . . . . . . . . . . . 6

5. Hagedorn Divergence . . . . . . . . . . . . . . . . . . . . . . . . . . . . . . 7

\section{Introduction}

An s-brane (spacelike-brane) is a topological defect all of whose longitudinal dimensions are spacelike and therefore exists only for a moment in time. The topological "scharge" is typically defined by an integral over a sphere which has both timelike and spacelike directions. In [1] it was argued that string theory contains s-branes. (See also [2 9].) The argument invoked the picture [10] of stable Dp-branes as domain walls spatially interpolating between two minima of the tachyon potential of unstable $\mathrm{D}(\mathrm{p}+1)$-branes. Sp-branes are related topological configurations in which the interpolation occurs in the time, rather than space, direction. Energy conservation requires both incoming and outgoing matter/radiation of some kind. An sp-brane can be described as a process in which incoming matter/radiation excites the tachyon field up to the top of the potential, from where it subsequently decays into the next minimum.

S-branes provide an interesting and relatively simple arena in which to study timedependent processes in string theory. In the large $\mathrm{N}$ limit, with $\mathrm{N}$ the number of S-branes, they may be holographically dual to interesting closed string cosmologies.

Recently Sen [11-14] has argued that a worldsheet boundary interaction sinh $X^{0}$, where $X^{0}$ is the timelike coordinate, gives an exact conformally invariant boundary sinh-Gordon type field theory and constructed the corresponding boundary state. This amounts to a CFT construction of the s-brane in a formal $g_{s}=0$ limit, in which quantum effects and closed strings are suppressed. It may be checked from the RR boundary state guessed in [13] that the s-brane indeed carries the required RR s-charge [15].

This talk we will discuss the related $e^{X^{0}}$ interaction, which is a kind of boundary Liouville theory for negative norm bosons. (Ordinary boundary Liouville theories have been studied in $16-18$. $)$ This is formally a limit of the $\sinh \left(X^{0}-a\right)$ type theory in which 
the location $a$ of the s-brane is taken to past infinity while scaling the interaction strength. It describes only the future, decaying, half of the solution, or a half-s-brane.

All of these solutions have the property, as shown in [11], that in the far future, after the decay is completed, the energy of the s-brane is converted into a pressureless tachyon dust which remains confined to the unstable $\mathrm{D}(\mathrm{p}+1)$-brane worldvolume. This tachyon dust is somewhat bizarre because the tachyon has reverted to its minimum and there should be no open string excitations. Presumably this picture is greatly modified for $g_{s} \neq 0$ [9]. Away from $g_{s}=0$, one expects that the energy should leak off the brane in the form of closed string radiation.

This talk will report on work in progress analyzing the decay process in bosonic string theory for $g_{s} \neq 0$. As a first step, in order to get a picture of what to expect, an adaptation of the 'mini-superspace' approximation of [19] (see also [20]) is employed. This approximation has proven generally useful for ordinary Liouville theory, but its validity has not been demonstrated in the present context. As the problem considered here is comparable in complexity to those solved in [16 [18], a more systematic analysis should be possible. We hope to report on this in the near future [21].

In the minisuperspace approximation all open string states acquire exponentially growing masses from the tachyon background. This results in open string pair production. We will find that for any nonzero $g_{s}$, the pair production is typically divergent 1 and the theory becomes strongly coupled in a time of order $\left(g_{s}\right)^{0}$. Roughly speaking what happens is that the decaying brane tries to produce open strings at the Hagedorn temperature. Whether or not this behavior survives the minisuperspace approximation remains to be seen [21]. This suggests that the $g_{s} \rightarrow 0$ limit is not smooth. A divergent density of open strings (with exponentially growing masses) will couple strongly to closed strings, suggesting that the s-brane energy is quickly released into closed strings. However string perturbation theory probably cannot be used to follow this process all the way through because of the intermediate strong coupling region.

The paper is organized as follows. In section 2 we compute the particle creation rate for a scalar field with an exponentially growing mass. In section 3 we show that in the minisuperspace approximation the effect of a time-dependent background tachyon is to give time-dependent masses to the open string modes. In section 4 we give the description

1 The divergence can apparently be suppressed for large numbers of noncompact transverse dimensions [22], as discussed in the last section. 
of the half-s-brane as a boundary Liouville theory. In section 5 we put this together and estimate the total perturbative open string creation rate. We find that it typically diverges due to the large number of states at high energy. Implications are discussed.

\section{Scalar Field with Time-Dependent Mass}

Consider the Klein-Gordon equation for a complex scalar with a time-dependent mass

$$
\begin{aligned}
& \left(-\partial_{t}^{2}+\nabla^{2}\right) \phi=m^{2}(t) \phi \\
& -\partial_{t}^{2} \phi=\left(m^{2}(t)+\vec{p}^{2}\right) \phi
\end{aligned}
$$

For sufficiently slowly varying $m$ the solutions behave as

$$
\phi \sim e^{ \pm i \sqrt{m^{2}(t)+\vec{p}^{2}} t}
$$

and hence oscillate more rapidly as $m$ is increased. The Klein-Gordon current

$$
j_{a}=i\left(\phi^{*} \partial_{a} \phi-\phi \partial_{a} \phi^{*}\right)
$$

is conserved for any $m(t)$. However since the mass is time dependent energy will not be conserved: raising the mass requires an input of energy.

\subsection{Exponential Mass}

Consider the special case

$$
m^{2}(t)=e^{2 t}+m_{0}^{2}
$$

We shall see that this is the typical behavior of an open string mass in a half-s-brane background: the mass goes to infinity for $t \rightarrow \infty$ as the tachyon decays. The solutions of (2.2) with (2.5) are

$$
\psi_{\vec{p}}^{i n}=\frac{2^{-i \omega}}{\sqrt{2 \omega}} \Gamma(1-i \omega) e^{i \vec{p} \cdot \vec{x}} J_{-i \omega}\left(e^{t}\right), \quad \omega \equiv \sqrt{m_{0}^{2}+\vec{p}^{2}}
$$

and its complex conjugate. These are normalized so that

$$
j_{0}(\vec{x}, t)=1 .
$$

In the far past this solution approaches a positive frequency plane wave

$$
t \rightarrow-\infty, \quad \psi_{\vec{p}}^{i n} \rightarrow \frac{1}{\sqrt{2 \omega}} e^{-i \omega t+i \vec{p} \cdot \vec{x}} .
$$

In the far future

$$
t \rightarrow \infty, \quad \psi_{\vec{p}}^{i n} \rightarrow \frac{2^{-i \omega} \Gamma(1-i \omega)}{\sqrt{\pi \omega}} e^{-t / 2+i \vec{p} \cdot \vec{x}} \cosh \left(\frac{\pi \omega}{2}-i e^{t}+i \frac{\pi}{4}\right) .
$$

Note that the frequency increases exponentially, corresponding to the higher energy, while the amplitude decreases. 


\subsection{Hyperbolic Mass}

Another relevant special case is $m^{2}(t)=\cosh 2 t$ :

$$
\partial_{t}^{2} \phi+\left(\cosh 2 t+\vec{p}^{2}\right) \phi=0
$$

This arises for open strings in a full s-brane, which are very massive in both the far past and the far future. The solution to this equation is a Mathieu function with an imaginary argument, which for large values of the argument approaches a Bessel function.

\subsection{Particle Creation}

According to (2.9), for an exponentially growing mass, the incoming modes $\psi_{\vec{p}}^{\text {in }}$ contain both negative and positive frequency parts in the far future. This indicates particle production. Normalized outgoing positive frequency modes are Hankel functions

$$
\psi_{\vec{p}}^{\text {out }}=\sqrt{\frac{\pi}{4 i}} e^{-\frac{\pi \omega}{2}+i \vec{p} \cdot \vec{x}} H_{-i \omega}^{(2)}\left(e^{t}\right) \rightarrow \frac{1}{\sqrt{2}} e^{-\frac{t}{2}-i e^{t}+i \vec{p} \cdot \vec{x}}, \quad t \rightarrow \infty
$$

The energy of these modes in the far future goes as

$$
\mathcal{E}(t)=\dot{\psi}_{\vec{p}}^{\text {out } *} \dot{\psi}_{\vec{p}}^{\text {out }}+\left(\omega^{2}+e^{2 t}\right) \psi_{\vec{p}}^{\text {out } *} \psi_{\vec{p}}^{\text {out }} \sim e^{t} \sim m(t)
$$

The energy grows with time because the equation of motion is time dependent. Using

$$
\begin{aligned}
H_{-i \omega}^{(2)}(x) & =\frac{1}{\sinh \pi \omega}\left(e^{\pi \omega} J_{-i \omega}(x)-J_{i \omega}(x)\right), \\
J_{i \omega}^{*}(x) & =J_{-i \omega}(x), \\
J_{-i \omega}(x) & =\frac{1}{2}\left(H_{-i \omega}^{(2)}(x)+H_{i \omega}^{(2) *}(x)\right), \\
|\Gamma(1+i \omega)|^{2} & =\frac{\pi \omega}{\sinh \pi \omega},
\end{aligned}
$$

for real $x, \omega$, we find

$$
\begin{aligned}
\psi_{\vec{p}}^{\text {out }} & =\alpha_{\vec{p}} \psi_{\vec{p}}^{i n}+\beta_{\vec{p}} \psi_{-\vec{p}}^{i n *}, \\
\psi_{\vec{p}}^{\text {in }} & =\alpha_{\vec{p}}^{*} \psi_{\vec{p}}^{\text {out }}-\beta_{\vec{p}} \psi_{-\vec{p}}^{\text {out } *}, \\
\alpha_{\vec{p}} & =\frac{2^{i \omega}}{\sqrt{2 \pi i \omega}} \Gamma(1+i \omega) e^{\frac{\pi \omega}{2}}, \\
\beta_{\vec{p}} & =-\frac{2^{-i \omega}}{\sqrt{2 \pi i \omega}} \Gamma(1-i \omega) e^{-\frac{\pi \omega}{2}} .
\end{aligned}
$$


Note that these Bogolubov coefficients obey $\alpha_{\vec{p}} \alpha_{\vec{p}}^{*}-\beta_{\vec{p}} \beta_{\vec{p}}^{*}=1$ as required. For large $\omega, \beta_{\vec{p}}$ approaches zero while $\alpha_{\vec{p}}$ becomes a pure phase. Expanding

$$
\phi=\sum_{\vec{p}}\left(\psi_{\vec{p}}^{i n} a_{\vec{p}}^{i n}+\psi_{\vec{p}}^{i n *} a_{\vec{p}}^{i n \dagger}\right)=\sum_{\vec{p}}\left(\psi_{\vec{p}}^{o u t} a_{\vec{p}}^{\text {out }}+\psi_{\vec{p}}^{\text {out } *} a_{\vec{p}}^{\text {out } \dagger}\right)
$$

the in vacuum becomes

$$
\mid \text { in }>=\prod_{\vec{p}}\left(1-\left|\gamma_{\vec{p}}\right|^{2}\right)^{1 / 4} e^{-\frac{1}{2} \sum \gamma_{\vec{p}}\left(a_{\vec{p}}^{\text {out } \dagger}\right)^{2}} \mid \text { out }>,
$$

where

$$
\gamma_{\vec{p}}=\frac{\beta_{\vec{p}}^{*}}{\alpha_{\vec{p}}}=-i e^{-\pi \omega}
$$

The in vacuum is annihilated by $a_{\vec{p}}^{i n}=\alpha_{\vec{p}} a_{\vec{p}}^{\text {out }}+\beta_{\vec{p}}^{*} a_{-\vec{p}}^{\text {out }}$.

Relation (2.16) expresses the fact that if there are no incoming particles at $t \rightarrow-\infty$, there will necessarily be outgoing particles at $t \rightarrow \infty$. The expected total number of particles created in each mode $\vec{p}$ is finite and largely occurs in a neighborhood of $t=0$. The creation is exponentially suppressed at frequencies $\omega>>\frac{1}{\pi}$.

\section{Minisuperspace Approximation}

In this section we consider the quantization of an open string in the half-s-brane using the minisuperspace approximation for the zero mode of $X^{0}$. This follows analogous treatments for the ordinary bulk Liouville theory in [19,20].

The bosonic part of the worldsheet action for an open string on an unstable bosonic D-brane is

$$
-\frac{1}{4 \pi \alpha^{\prime}} \int d \tau d \sigma \sqrt{-\gamma} \gamma^{a b} \partial_{a} X^{\mu} \partial_{b} X_{\mu}-\frac{1}{8 \pi} \int d \tau \sqrt{-h} T(X)
$$

where $T$ is the background tachyon field, $0 \leq \sigma \leq \pi$ and $h$ is the boundary metric induced from the worldsheet metric $\gamma$. The second integral runs over all boundaries of the worldsheet. For the superstring $T$ is replaced by $T^{2}$ [10,23], ensuring that the potential is bounded from below for all background tachyon fields.

In a minisuperspace type approximation we treat the zero mode as independent of the oscillators. We wish to consider the zero mode behavior for the case $T=T\left(X^{0}\right)$. The pure zero mode piece of (3.1) for $\gamma=\eta$ is (for $X^{\mu}$ independent of $\sigma$ )

$$
S_{0}=\frac{1}{4 \alpha^{\prime}} \int d \tau\left(-\left(\dot{X}^{0}\right)^{2}+\left(\dot{X}^{i}\right)^{2}-\frac{\alpha^{\prime} T\left(X^{0}\right)}{\pi}\right) .
$$


The corresponding zero mode Hamiltonian is

$$
H_{0}=-\alpha^{\prime}\left(P^{0}\right)^{2}+\alpha^{\prime}\left(P^{i}\right)^{2}+\frac{T\left(X^{0}\right)}{4 \pi} .
$$

Including oscillator contributions the constraint $L_{0}+\bar{L}_{0}=0$ becomes the Schroedinger equation for the open string wave function

$$
\left(\frac{\partial^{2}}{\partial X^{02}}+\frac{T\left(X^{0}\right)}{4 \pi \alpha^{\prime}}+\frac{N-1}{\alpha^{\prime}}+p^{i} p^{i}\right) \psi\left(X^{0}\right)=0
$$

Here $p^{i}$ are the constants of motion corresponding to the zero mode momenta $P^{i}$ in (3.3) and $N$ are the "oscillator" contributions.

Making the identification $t \rightarrow X^{0}$ and

$$
m^{2}\left(X^{0}\right)+\vec{p}^{2}=\frac{T\left(X^{0}\right)}{4 \pi \alpha^{\prime}}+\frac{N-1}{\alpha^{\prime}}+p^{i} p^{i}
$$

(3.4) is in fact exactly the wave equation (2.2) for a scalar field with time-dependent mass. This might have been anticipated. The worldsheet tachyon interaction is represented by the proper length of the end of the string worldsheet weighted by the background tachyon. This is equivalent to hanging masses at the ends of the string proportional to $T\left(X^{0}\right)$. Therefore a time dependent tachyon gives a time-dependent mass to all open string states. In the limit of $T \rightarrow \infty$ one expects to recover the closed string vacuum. Indeed in this limit all open string masses go to infinity.

\section{The Half-s-brane and Boundary Liouville Theory}

In order that (3.1) describe a consistent open string background $T$ should be a conformally invariant interaction. We consider the case

$$
T=e^{X^{0} / \sqrt{\alpha^{\prime}}}
$$

This is a dimension one operator because the $X^{0}$ has the "wrong sign" two-point function. (4.1) defines a boundary Liouville theory, which has been studied (for a positive norm field) in 16-18. In the past $X^{0} \rightarrow-\infty$, the tachyon field is perched at the top of its potential. It falls off towards the future, and reaches the closed string vacuum in the far future, where all open strings become infinitely massive. It describes the decay of an unstable brane or, equivalently, the future half of an s-brane. 
The open string spectrum is of course continuous because of the noncompact region. In the usual (positive norm) boundary Liouville theory there is a non-trivial density of states which is related in [17] to the reflection coefficients off of the tachyon wall, which in turn are related to the two point function on the disc. In our case however, there is no reflection simply because the potential implied by (4.1) has a negative sign relative to the $X^{0}$ kinetic term, and both solutions are allowed in the future. Hence the density of states is the same as it is for free field theory.

This has a simple interpretation. The open string spectrum on the half s-brane can be computed in the far past $X^{0} \rightarrow-\infty$ where the effect of the tachyon is negligible. The $L_{0}$ eigenvalue does not depend on $X^{0}$ and so is the same in the far future. However the open string eigenfunctions are of course functions of $X^{0}$. In fact the effective open string masses appearing in (3.5) are of the exponential form (2.5)

$$
m^{2}\left(X^{0}\right)=m_{0}^{2}+\frac{e^{X^{0} / \sqrt{\alpha^{\prime}}}}{4 \pi \alpha^{\prime}}
$$

for $X^{0}=2 \sqrt{\alpha^{\prime}} t$. Hence the typical behavior of the open string eigenfunctions is expected to be of the form (2.6) with rapid oscillations in the far future.

\section{Hagedorn Divergence}

In this section we discuss open string pair production on a half-s-brane. The first step is to define the incoming state of the open string modes. Since the background tachyon field vanishes in the far past, it is natural to take all the incoming open string modes in their usual ground state. This does not work for the tachyon for the usual reason: there is no quantum ground state for a particle perched on top of a hill. If we try to force it to sit exactly at the maximum, it will have large fluctuations in its momentum. What this means is that in the quantum world the decay process will always begin some finite time after the initial conditions are set. In the following we presume that the creation of non-tachyonic open strings modes, whenever this decay process starts, is approximated by open string creation in the half-s-brane background.

The (expectation value of the) energy dumped by the decaying brane into open string pair production will contain factors such as

$$
\int N_{\omega} d \mathcal{E}_{\omega}
$$


The sum is over all single open string modes with incoming energy labelled by $\omega$ and density of states $N_{\omega} \cdot d \mathcal{E}_{\omega}$ is the (expectation value of the) outgoing energy in these modes, which may contain multiple quanta. Approximating the outgoing state by the right hand side of (2.16) and using (2.12) one finds for large $t$

$$
d \mathcal{E}_{\omega}=\frac{e^{2 \pi X^{0} T_{H}} d^{p} \vec{p}}{(2 \pi)^{p}\left(e^{\omega / T_{H}}-1\right)}, \quad T_{H}=\frac{1}{4 \pi \sqrt{\alpha^{\prime}}},
$$

where $p$ is the dimension of the s-brane. 2 At large $\omega[22]$

$$
N_{\omega} \sim \omega^{-a} e^{\omega / T_{H}}, \quad d \mathcal{E}_{\omega} \sim \omega^{p-1} e^{-\omega / T_{H}} e^{2 \pi X^{0} T_{H}} d \omega
$$

where the constant $a$ here is the number of non-compact directions transverse to the brane. Evidently the integral (5.1) diverges, except for sufficiently large numbers of non-compact

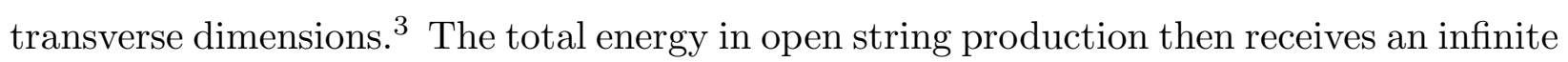
contribution from high-energy modes. Roughly speaking the brane tries to produce open strings at the Hagedorn temperature (although the final state is of course a pure state). Since the particle production turns on slowly, there must be some finite time $t_{C}$ at which the production rate diverges. $t_{C}$ is independent of $g_{s}$.

Of course when back reaction is included, this is forbidden by energy conservation. The energy for the pair production must come out of the the tachyon background. No matter how small $g_{s}$ is the open strings become strongly coupled before $t=t_{C}$ and the linearized approximation breaks down. Eventually all the energy goes into closed strings. It is hard to say exactly how this occurs, but the divergence makes it plausible that the brane energy is dumped into closed strings in a time of order $t_{C}$, despite the fact that closed strings formally decouple for $g_{s} \rightarrow 0$. In any case because of the divergence, the formal $g_{s} \rightarrow 0$ limit appears not to be smooth in the minisuperspace approximation.

\section{Acknowledgements}

I am grateful to M. Gutperle, A. Maloney, S. Minwalla, A. Recknagel, V. Schomerus, A. Sen and S. Shenker for useful conversations. This work was supported in part by DOE grant DE-FG02-91ER40654.

2 Frequencies here are defined relative to $X^{0}=2 \sqrt{\alpha^{\prime}} t$.

3 This is analogous to 'limiting' and 'non-limiting' Hagedorn behavior [22].

4 Conceivably this is related to the time at which the tachyon becomes massless. 


\section{References}

[1] M. Gutperle and A. Strominger, "Spacelike branes," JHEP 0204, 018 (2002) hepth/0202210.

[2] C. M. Hull, "Timelike T-duality, de Sitter space, large N gauge theories and topological field theory," JHEP 9807, 021 (1998) hep-th/9806146.

[3] H. Lu, S. Mukherji and C. N. Pope, "From p-branes to cosmology," Int. J. Mod. Phys. A 14, 4121 (1999) hep-th/9612224.

[4] H. Lu, S. Mukherji, C. N. Pope and K. W. Xu, "Cosmological solutions in string theories," Phys. Rev. D 55, 7926 (1997), hep-th/9610107.

[5] C. M. Chen, D. V. Gal'tsov and M. Gutperle, "S-brane solutions in supergravity theories," Phys. Rev. D 66, 024043 (2002), hep-th/0204071.

[6] M. Kruczenski, R. C. Myers and A. W. Peet, "Supergravity S-branes," JHEP 0205, 039 (2002), hep-th/0204144.

[7] N. S. Deger and A. Kaya, "Intersecting S-brane solutions of D = 11 supergravity," JHEP 0207, 038 (2002), hep-th/0206057.

[8] S. Roy, "On supergravity solutions of space-like Dp-branes," JHEP 0208, 025 (2002), hep-th/0205198.

[9] T. Okuda and S. Sugimoto, "Coupling of rolling tachyon to closed strings," hepth/0208196.

[10] A. Sen, "Non-BPS states and branes in string theory," hep-th/9904207.

[11] A. Sen, "Rolling tachyon," JHEP 0204, 048 (2002) hep-th/0203211.

[12] A. Sen, "Tachyon matter," hep-th/0203265.

[13] A. Sen, "Field theory of tachyon matter," hep-th/0204143.

[14] A. Sen, "Time evolution in open string theory," hep-th/0207105.

[15] M. Gutperle and A. Strominger, unpublished.

[16] V. Fateev, A. B. Zamolodchikov and A. B. Zamolodchikov, "Boundary Liouville field theory. I: Boundary state and boundary two-point function," hep-th/0001012.

[17] J. Teschner, "Remarks on Liouville theory with boundary," hep-th/0009138.

[18] B. Ponsot and J. Teschner, "Boundary Liouville field theory: Boundary three point function," Nucl. Phys. B 622, 309 (2002) hep-th/0110244.

[19] E. Braaten, T. Curtright, G. Ghandour and C. B. Thorn, "Nonperturbative Weak Coupling Analysis Of The Liouville Quantum Field Theory," Phys. Rev. Lett. 51, 19 (1983); Annals Phys. 153, 147 (1984).

[20] J. Polchinski, "Remarks On The Liouville Field Theory," UTTG-19-90 Presented at Strings '90 Conf., College Station, TX, Mar 12-17, 1990.

[21] M. Gutperle, A. Recknagel, V. Schomerus and A. Strominger, in progress.

[22] See e.g. S. A. Abel, J. L. Barbon, I. I. Kogan and E. Rabinovici, "String thermodynamics in D-brane backgrounds," JHEP 9904, 015 (1999) hep-th/9902058.

[23] D. Kutasov, M. Marino and G. W. Moore, "Remarks on tachyon condensation in superstring field theory," hep-th/0010108. 